\title{
TrawlerWeb: an online de novo motif discovery tool for next-generation sequencing datasets
}

Louis T. Dang ${ }^{1}$, Markus Tondl ${ }^{1}$, Man Ho H. Chiu', Jerico Revote², Benedict Paten ${ }^{3}$, Vincent Tano ${ }^{4}$, Alex Tokolyi ${ }^{1}$, Florence Besse ${ }^{5}$, Greg Quaife-Ryann ${ }^{6}$, Helen Cumming ${ }^{7}$, Mark J. Drvodelic ${ }^{1}$, Michael P. Eichenlaub ${ }^{1}$,

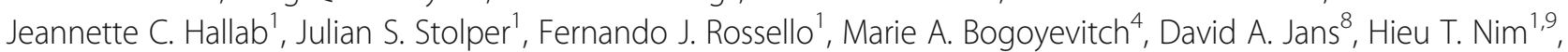
Enzo R. Porrello ${ }^{10,11}$, James E. Hudson ${ }^{6}$ and Mirana Ramialison ${ }^{1 *}$

\begin{abstract}
Background: A strong focus of the post-genomic era is mining of the non-coding regulatory genome in order to unravel the function of regulatory elements that coordinate gene expression (Nat 489:57-74, 2012; Nat 507:462-70, 2014; Nat 507:455-61, 2014; Nat 518:317-30, 2015). Whole-genome approaches based on next-generation sequencing (NGS) have provided insight into the genomic location of regulatory elements throughout different cell types, organs and organisms. These technologies are now widespread and commonly used in laboratories from various fields of research. This highlights the need for fast and user-friendly software tools dedicated to extracting cis-regulatory information contained in these regulatory regions; for instance transcription factor binding site (TFBS) composition. Ideally, such tools should not require prior programming knowledge to ensure they are accessible for all users.

Results: We present TrawlerWeb, a web-based version of the Trawler_standalone tool (Nat Methods 4:563-5, 2007; Nat Protoc 5:323-34, 2010), to allow for the identification of enriched motifs in DNA sequences obtained from nextgeneration sequencing experiments in order to predict their TFBS composition. TrawlerWeb is designed for online queries with standard options common to web-based motif discovery tools. In addition, TrawlerWeb provides three unique new features: 1) TrawlerWeb allows the input of BED files directly generated from NGS experiments, 2) it automatically generates an input-matched biologically relevant background, and 3) it displays resulting conservation scores for each instance of the motif found in the input sequences, which assists the researcher in prioritising the motifs to validate experimentally. Finally, to date, this web-based version of Trawler_standalone remains the fastest online de novo motif discovery tool compared to other popular web-based software, while generating predictions with high accuracy.

Conclusions: TrawlerWeb provides users with a fast, simple and easy-to-use web interface for de novo motif discovery. This will assist in rapidly analysing NGS datasets that are now being routinely generated. TrawlerWeb is freely available and accessible at: http://trawler.erc.monash.edu.au.
\end{abstract}

Keywords: Motif discovery, Transcription factor binding site, Motif conservation, Chromatin immunoprecipitation, Next generation sequencing

\footnotetext{
* Correspondence: mirana.ramialison@monash.edu

${ }^{1}$ Australian Regenerative Medicine Institute, Systems Biology Institute

Australia, Monash University, Clayton, VIC, Australia

Full list of author information is available at the end of the article
}

(c) The Author(s). 2018 Open Access This article is distributed under the terms of the Creative Commons Attribution 4.0 International License (http://creativecommons.org/licenses/by/4.0/), which permits unrestricted use, distribution, and reproduction in any medium, provided you give appropriate credit to the original author(s) and the source, provide a link to the Creative Commons license, and indicate if changes were made. The Creative Commons Public Domain Dedication waiver (http://creativecommons.org/publicdomain/zero/1.0/) applies to the data made available in this article, unless otherwise stated. 


\section{Background}

Understanding how genes are regulated is the challenge of the post-genomic era [1-4]. The ability to identify cisregulatory combinations $[5,6]$ that affect the spatiotemporal control of gene expression is important in elucidating disease and developmental processes [7, 8]. Genome-wide binding assays such as chromatin immunoprecipitation (ChIP) [9], DNA adenine methyltransferase identification (DamID) [10, 11], and transposase-accessible chromatin (ATAC) [12] coupled with next-generation sequencing (NGS) technologies are popular methods to uncover regulatory regions that will shed light on our understanding of gene regulation $[13,14]$. These methods have been coopted amongst scientists working on model organisms ranging from plants to humans $[15,16]$. The downstream analysis of these genome-wide assays consists of identifying specific binding motifs in DNA, which ultimately encode for regulatory information. To achieve this, several motif discovery algorithms have been generated to identify specific binding motifs using different algorithms, such as exhaustive pattern-based enumeration, variants of Markov chain Monte Carlo (MCMC) or deep learning models [17-23].

As NGS technologies are now routinely used in all laboratories, with the emergence of more online platforms for NGS data processing e.g. Galaxy [24], ChIP-seq tools [25], there is a need to streamline the motif discovery process, ideally with the convenience of web-based interfaces accepting input queries. Accurate binding site prediction, speed and ease-of-use are key criteria for users when selecting a preferred motif discovery tool. Finally, selecting the motifs to be experimentally tested amongst the list of predicted motifs still represents a challenge.

Two of the most cited tools for de novo motif discovery, MEME-ChIP and RSAT peak-motifs provide a userfriendly interface and have been used to successfully identify transcription factor binding sites $[18,20]$. DeepSEA also offers an online web search interface, but input sequences are currently limited to 1000 base pairs (bp) and only queries against the Human Genome (hg19) [22]. Trawler_standalone is one of the fastest motif discovery tools available, while still providing accurate predictions [5], however it is currently only available as a command-line standalone version [6]. Here we present TrawlerWeb, which streamlines de novo motif discovery with NGS datasets from a wide range of species. This web-based version provides three new unique features that allow it to streamline and facilitate the analysis of predicted motifs: 1) in addition to FASTA-formatted sequences, it accepts direct input from ChIP-seq experiments in BED format, 2) it automatically generates a set of background sequences matching the input sequences in terms of genomic location and 3) it allows the ranking of predicted motifs by conservation score to select those more suited for downstream experimental validation. After systematically comparing TrawlerWeb with the web versions of MEME-ChIP and RSAT peak-motifs, we demonstrated that in accordance with Trawler_standalone performance [5], TrawlerWeb still remains the fastest online motif discovery tool while maintaining motif prediction accuracy.

\section{Implementation \\ Web implementation}

TrawlerWeb is running on a standard Apache web server configuration under a Linux environment. It has been deployed and supported on the Monash node (R@CMon) of the Nectar Research Cloud. TrawlerWeb has been rigorously tested by a total of 11 different users on five different datasets using Chrome, Firefox and Internet Explorer web browsers with Linux, Windows and Mac OS X operating systems (Table 1).

\section{Input file}

The web interface for TrawlerWeb offers users the option of uploading input sequences as BED indexed format, in addition to FASTA files, which is the most common form of input offered by most motif discovery tools. BED files are lists of genomic intervals and are the direct output from ChIP-seq experiments. Users can therefore directly input the data of their ChIP-seq experiments in TrawlerWeb, without having to retrieve corresponding FASTA sequences. Note that PSCAN [21] also offers BED file input but performs motif discovery on known motifs rather than de novo. DeepSEA [22] accepts input files as BED format, however, currently only one genome (hg19) is supported and input sequence length is limited to $1000 \mathrm{bp}$. Since peaks obtained from ChIP-seq on histone modifications, for example, could exceed $1000 \mathrm{bp}$, this option might be restrictive to users. At present, TrawlerWeb remains the only online motif

Table 1 Operating systems and browsers on which 11 users have successfully tested TrawlerWeb

\begin{tabular}{lll}
\hline User & Operating System & Used browser \\
\hline 001 & MAC OS X 10.11 & Mozilla Firefox \\
002 & Windows 10 & Google Chrome \\
003 & MAC OS X 10.11.6 & Mozilla Firefox \\
004 & Windows 8.1 & Google Chrome \\
005 & MAC OS X 10.10 & Mozilla Firefox \\
006 & Linux Ubuntu 16.04 & Mozilla Firefox \\
007 & Windows 7 Enterprise & Mozilla Firefox \\
008 & MAC OS X 10.11 & Google Chrome \\
009 & MAC OS X 10.9.5 & Google Chrome \\
010 & Windows 7 Enterprise & Internet Explorer \\
011 & MAC OS X 10.9.5 & Google Chrome \\
\hline
\end{tabular}


discovery tool accepting input files in BED format for a large range of species.

Regions can be either uploaded as BED files or directly pasted into the website, with users required to select the genome assembly of their desired model organism. To date, TrawlerWeb supports 16 genome assemblies (Table 2), downloaded from EnsEMBL [26]. To streamline analysis of data generated from ChIP-seq experiments and other DNA-binding assays, FASTA sequences corresponding to the input BED regions are automatically extracted from locally stored chromosome assemblies. Prior to retrieving FASTA sequences, the BED regions are processed using BEDtools' merge tool with default settings to avoid duplicated locations [27]. FASTA sequences are repeat-masked to prevent the discovery of repetitive motifs produced from lowcomplexity and repeat regions [5]. Users also have the option of using non-masked sequences if the immunoprecipitated transcription factor is known or hypothesised to bind to repeat sequences [28].

\section{Background file}

For each set of input BED regions, a randomised background specific to the dataset is automatically generated to match the distribution of the genomic locations of the input regions. This input-matched background allows TrawlerWeb to account for sequence biases present in certain genomic regions (for example promoter regions are known to be enriched in CpG islands [29]).

To generate a customised background dataset, first, TrawlerWeb calculates the distribution of the distances

Table 2 Species and genome assemblies supported by TrawlerWeb

\begin{tabular}{lll}
\hline & Species & Genome assemblies \\
\hline Fish & Oryzias latipes & oryLat2 \\
Zebrafish & Danio rerio & danRer7 \\
Stickleback & Gasterosteus aculeatus & gasAcu1 \\
Tetrapods & & \\
Human & Homo sapiens & hg19, hg38 \\
Mouse & Mus musculus & mm9, mm10 \\
Rat & Rattus norvegicus & rn5 \\
Marmoset & Callithrix jacchus & calJac3 \\
Chicken & Gallus gallus & galGal3 \\
Clawed frog & Xenopus tropicalis & xenTro3 \\
Other eukaryotes & & \\
Fruit fly & Drosophila melanogaster & $\mathrm{dm} 3, \mathrm{dm6}$ \\
Worm & Caenorhabditis elegans & ce10 \\
Yeast & Saccharomyces cerevisiae & sacCer3 \\
Thale cress & Arabidopsis thaliana & TAlR9 \\
\hline
\end{tabular}

of the input regions with respect to the nearest transcription start site (TSS). Each input region is associated to a 'nearest gene' and the distance of this region to the gene's TSS is calculated using gene coordinates downloaded from EnsEMBL using BioMART [26, 30] for the given organism. Distances are then plotted across discrete ranges (e.g. $-5000 \mathrm{bp}$ to 0,0 to $5000 \mathrm{bp}$, etc.) to produce a frequency table representing the input regions. Next, genes are randomly selected from the entire genome and genomic regions are extracted upstream or downstream of the TSS, so that the distribution of the selected regions match the frequency table generated for input regions. This frequency table is displayed on the results page as TrawlerWeb is running. The amount of randomly selected regions to generate this background dataset is eight times the amount of regions in the input, which we have previously demonstrated to robustly provide adequate background dataset $[5,6]$.

As a new background will be generated for each new input submission, users have the option to download the background FASTA sequences generated for a specific FASTA input, should they need to re-run the exact same analysis using the same background.

\section{Input options}

TrawlerWeb comes with an array of options to optimise the user's search results. By default, Trawler will search for motifs that are at least $8 \mathrm{bp}$ in length and at most $20 \mathrm{bp}$. However, the user can reduce the minimum motif length in order to allow motifs of shorter length to be identified.

The wildcard option allows for mismatches in the identified motif. For a minimum motif length of 8 , two wildcards are used by default. Should the user choose to reduce the minimum motif length (e.g. 6), one wildcard is recommended to maintain sensitivity.

If the final list of clustered motifs (named families) in the results page retain some similarity and should be clustered together, the "percentage overlap" option can be reduced so that the amount of similarity required between instances to be clustered is reduced.

The frequency at which a motif is expected to occur in a sample can vary depending on the type of data. Generally, a minimum of 10 to 20 occurrences is suitable for most ChIP experiments. If this parameter is set too low, Trawler will be unable to identify any significant results whereas setting the parameter too high when the desired motif is not present in all sample sequences will produce a motif with low information content. This can be configured using the "occurrence" option.

The number of motifs used for clustering is determined by the "number of motifs" option. By default, Trawler takes the top 200 ranked motifs for clustering. To allow for identification of secondary and possibly 
tertiary motifs, the number of motifs can be increased, however this will also increase computation time.

After running Trawler initially with default settings once, the final number of motifs identified can be finetuned by indicating a value for "number of clusters" option. By default, Trawler will cluster by strongly connected component (SCC) when "number of clusters" is set to zero. However, k-means clustering can be used to cluster the motifs into $\mathrm{n}$ clusters by providing an integer value $(\mathrm{n})$ to this option.

\section{Output files and data download}

The final results are summarised as a web page displaying all discovered motifs in a table along with $\mathrm{z}$ scores for statistical over-representation [5]. Putative matches against known TFBSs and maximum sequence conservation of the motif's instances will be displayed in this summary web page. By default, motifs are ordered in decreasing order of conservation score, however any of the columns can be used for sorting the output. For each identified motif, further information is available upon clicking on the motif name of logo. First, the distribution of the motif locations within the input sequences can be visualised as a histogram. Consensus sequence, length and identity of the TFBS match are displayed in the next table where a mouse-over on the column titles will provide a description of the properties of the match. A link to the original TFBS will allow to visualise the putative hit from either UniPROBE, Jaspar and HOCOMOCO databases [31-34]. The final table lists the location of every instance of the predicted motif in the input sequences, along with an average and maximum conservation score within the instance. Each location is linked to a dynamic view of the region in the UCSC genome browser [35]. Similarly, a mouseover on the column's title will provide further information. All tables in this detailed page are sortable by column, searchable and are dynamically filtered for the searched term. The entire results webpage and files generated by TrawlerWeb are also available for download in a single zip file.

\section{Results and discussion}

TrawlerWeb runs the fastest amongst popular web-based motif discovery tools

We aimed to compare the performance of TrawlerWeb with the popular web-based de novo motif discovery tools RSAT peak-motifs [20, 36] and MEME-ChIP [18]. For this, 11 users were given five different ChIPseq datasets from five commonly used model organisms in FASTA format (Table 3). The same FASTA input file was used across the three different programs with the same background file used for TrawlerWeb and RSAT peak-motifs, default background was used for MEME-ChIP as it does not allow for custom background FASTA input. All other options were kept as default. Running time was recorded from when the "Submit Query", "GO" or "Start Search" button was clicked for TrawlerWeb, RSAT peak-motifs and MEME-ChIP respectively, until the final list of motifs was returned. This also includes the queuing time which realistically reflects the actual waiting time experienced by users.

For all five species tested (Table 3), TrawlerWeb ran the fastest (Fig. 1a) in accordance with its standalone version $[5,6]$. TrawlerWeb generally returned the discovered motifs in less than $2 \mathrm{~min}$ ( $\mathrm{min}$ ) for the four smaller datasets Danio rerio (Dr), Arabidopsis thaliana (At), Mus musculus $(\mathrm{Mm})$ and Drosophila melanogaster $(D m)$. Only for the human dataset $(H s)$, being the largest input file tested, running time ranged from $4 \mathrm{~min}$ to over $21 \mathrm{~min}$, averaging $10 \mathrm{~min}$ overall. RSAT peak-motifs identified motifs after 210 min with only a few outliers. Motif discovery for the human dataset ran for 17 to $22 \mathrm{~min}$, however, one run was completed in under $7 \mathrm{~min}$. Nonetheless, we experienced larger variations in processing times with RSAT peak-motifs compared to TrawlerWeb. MEME-ChIP had the longest running time among the three tested tools. Typical motif discovery was completed on average after about $30 \mathrm{~min}$, with the exception of $H s$ which finished after up to almost $1 \mathrm{~h}$. Of note, MEME-ChIP provides by default two different algorithms, MEME and DREME. DREME [37] discovers short and ungapped motifs. Since MEME did

Table 3 ChIP-seq on transcription factors and genome assemblies used to compare TrawlerWeb, RSAT peak-motifs and MEME-ChIP

\begin{tabular}{lllllll}
$\begin{array}{l}\text { Transcription } \\
\text { factor }\end{array}$ & $\begin{array}{l}\text { ChIP-seq GEO } \\
\text { accession number }\end{array}$ & $\begin{array}{l}\text { Reference for } \\
\text { ChIP-seq }\end{array}$ & $\begin{array}{l}\text { ChIP-seq dataset } \\
\text { size (kbp) }\end{array}$ & $\begin{array}{l}\text { Reference for } \\
\text { known binding site }\end{array}$ & Species & Genome \\
\hline Zic3.2 & GSM1017643 & Winata et al., 2013 [49] & 282 & JASPAR PB0207.1 & D. rerio & danRer7 \\
TOC1 & GSM878068 & Huang et al., 2012 [47] & 343 & Huang et al., 2012 & A. thaliana & TAIR9 \\
MEF2A & GSM1377538 & Houles et al., 2015 [46] & 338 & JASPAR MA0052.3 & M. musculus & mm9 \\
Su(H) & GSE66225 & Skalska et al., 2015 [48] & 475 & JASPAR MA0085.1 & D. melano-gaster & dm3 \\
Sox15.1 & GSM1536045 & Sulahian et al., 2015 [40] & 1783 & JASPAR PB0065.1 & H. sapiens & hg19 \\
\hline
\end{tabular}




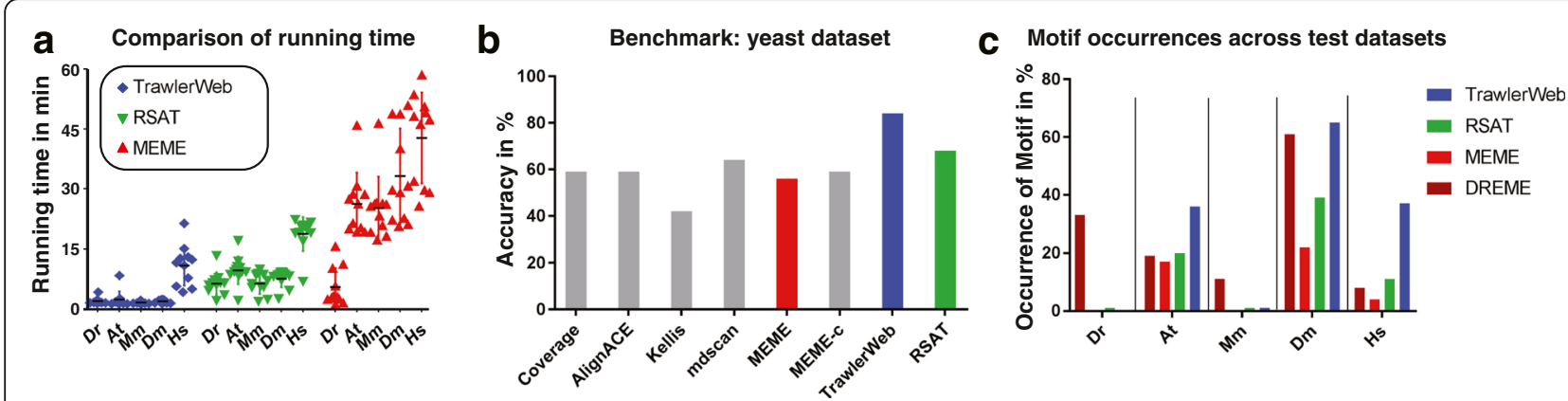

Fig. 1 Comparing the performance of TrawlerWeb with other web-based motif discovery tools. a Duration of 11 independent runs for TrawlerWeb (blue), RSAT peak-motifs (green) and MEME-ChIP (red) in minutes. The mean is represented by the horizontal line for each dataset. The error bars indicate the standard deviation from the mean. The data are ordered by increasing size of the FASTA input file from left to right. Note that MEME-ChIP did not find any motifs for Dr, hence the motif discovered by DREME was used (see also Fig. 2a). b Overall performance benchmark of TrawlerWeb against 7 other algorithms, using 65 ChIP pulled down experiments on yeast dataset from [38]. MEME-C: MEME algorithm run on conserved regions only. c Comparison of percentage occurrence of over-represented motifs across test datasets. Motif discovery were conducted using 4 algorithms (DREME, MEME, RSAT peak-motifs, and TrawlerWeb) on the test datasets and the number of sequences containing the highest scoring motif were expressed as a percentage of the total number of analysed input sequences. The MEME-ChIP pipeline uses both MEME and DREME motif discovery tools for finding relatively long and short motifs respectively. The MEME algorithm uses a random subsample of 600 sequences. Dr = Danio rerio, At = Arabidopsis thaliana, Mm = Mus musculus, Dm = Drosophila melanogaster, Hs = Homo sapiens

not find any motifs for the zebrafish dataset $(D r)$ we used the results provided by DREME, which can explain the shorter time compared to the performance of MEME.

In conclusion, in all tested cases, TrawlerWeb outperformed popular web-based de novo motif discovery tools in terms of speed.

TrawlerWeb accurately identifies the expected binding site To measure TrawlerWeb's accuracy against other online motif discovery tools, we tested TrawlerWeb against a yeast ChIP benchmark dataset [38]. This same dataset was previously used to test Trawler_standalone algorithm against other software [5, 6] but lacked RSAT peak-motifs [20], which we have included in this run. On this yeast dataset, TrawlerWeb outperformed all other methods in terms of accuracy, identifying 54 out of 65 motifs previously found (85\%; Fig. $1 \mathrm{~b}$ and Additional file 1: Table S1). These results were expected since TrawlerWeb and Trawler_standalone share the same underlying algorithm.

In order to further characterise TrawlerWeb's accuracy to identify the expected binding sites, we compared the motifs predicted by each tool, across eight users, to the known binding site of the transcription factor of interest, for the five ChIP-seq datasets (Table 3). To identify which predicted motif best matched the known binding site, all predicted motifs with the two highest z-scores (TrawlerWeb) or lowest e-values (RSAT peak-motifs, best of $6 \mathrm{nu}$ cleotide or 7 nucleotide length, and MEME/DREME) were aligned and clustered with the expected binding sites (Table 3) using STAMP (default options) [39]. For each ChIP-seq dataset, and for each program, motifs with the shortest distance to the expected binding site were identified as the closest motif. Across all users, the closest primary and secondary motifs (Fig. 2a-e), and corresponding distance to the expected binding site (Fig. 2f), were downloaded from STAMP in Newick format and visualised with iTOL using the expected motif as a reference. In two cases out of five, TrawlerWeb and RSAT predicted the known binding sites (for Zic3.2 (Fig. 2a), TOC1 (Fig. 2b)). TrawlerWeb is the only tested tool that identified the expected binding site for MEF2A (Fig. 2c), albeit as a secondary motif. In the case of $\mathrm{Su}(\mathrm{H})$ (Fig. 2d), TrawlerWeb and RSAT peak-motifs identified the same primary motif, which interestingly, is different to the expected binding site. Finally for Sox15.1 (Fig. 2e), RSAT peak-motifs discovered a similar motif to the known binding site, whereas TrawlerWeb found motifs that are quite different. However, these motifs resemble the PWM of Sox15.2 [32] suggesting that the reported binding site of Sox15 [40] is Sox15.2 rather than Sox15.1. In two out of five cases (Fig. 1b, d) the primary motifs discovered by MEMEChIP agreed with the other motif discovery tools and the expected binding site. For MEF2A and Sox15.1, the motifs identified by MEME-ChIP have low similarity to the known binding sites. For Zic3.2 MEME-ChIP did not find any motifs, hence we used DREME, which found two motifs that are quite distant from the expected binding site.

Of note, in three out of five datasets, TrawlerWeb identifies the predicted motif from the highest number of input sequences. When comparing over-represented motifs (based on the percentage of motif occurrences of the highest score) discovered using DREME, MEME, RSAT peakmotifs, and TrawlerWeb (Fig. 1c), TrawlerWeb returns 


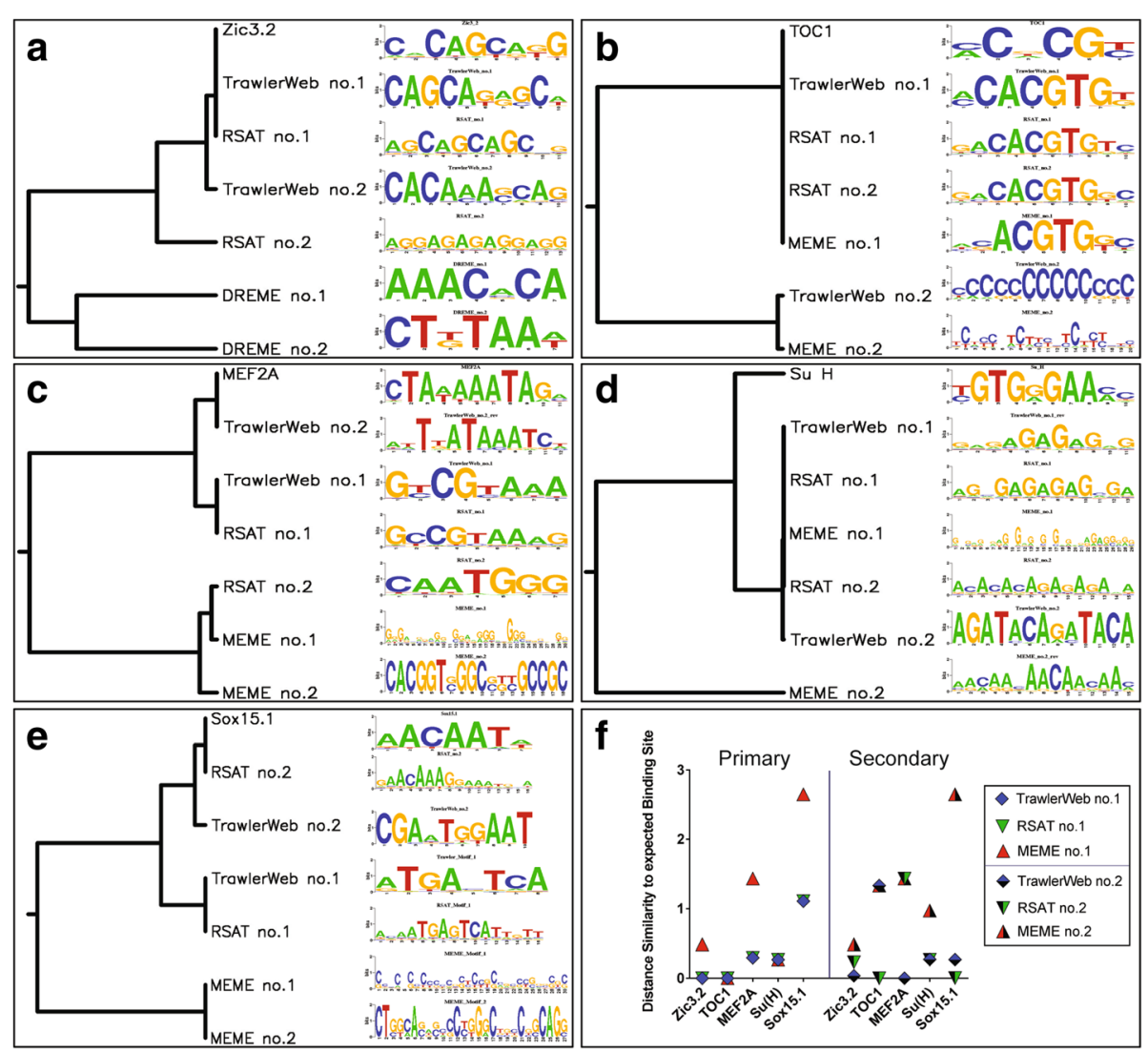

Fig. 2 Finding the expected motif with TrawlerWeb, RSAT peak-motifs, and MEME-ChIP. Alignment of the closest primary (no.1) and secondary (no.2) motif to the expected binding site identified for each motif discovery tool for the five species a Danio rerio, b Arabidopsis thaliana, c Mus musculus, d Drosophila melanogaster, and e Homo sapiens. f For each tool, Similarity Distance of the closest primary (no.1) and secondary (no.2) motif to the expected binding site. Motifs of 6 nucleotides (nt) length were represented for Su(H) and Sox15.1, and 7 nt for MEF2A, TOC1, and Zic3.2. MEME did not find any motif for Zic3.2, motif found by DREME was used

the highest percentage occurrence in $d m 3$, hg19 and tair 9 test datasets $(65.5 \%, 37.6 \%$ and $36.6 \%$ respectively), and delivers occurrences comparable to both MEME and RSAT peak-motifs in the $m m 9$ and $z v 9$ datasets $(1.6 \%$ and $0.3 \%$ ) (Additional file 2: Table S2). Variations in motif occurrence could be due to the presence of a secondary motif corresponding to a co-factor of the TF of interest [11]. Indeed, for TOC1, MEF2A and $\mathrm{Su}(\mathrm{H})$ datasets, TrawlerWeb identified a motif different to the known binding site (Fig. 2b-d). Overall, TrawlerWeb robustly identifies the primary motif with high similarity to the expected binding site (Fig. 2f).

\section{TrawlerWeb offers the unique option of displaying motif conservation scores}

Motif discovery tools deliver a list of over-represented putative DNA binding sites, usually ranked by overrepresentation score. Motifs are then often selected for experimental validation, for instance by verifying whether the transcription factor of interest effectively binds to the predicted motif. Amongst all of the instances of the predicted binding site in the submitted sequences, selecting the ones for experimental validation is not trivial. In order to prioritise identified motifs for downstream analysis, evolutionary conservation has been used as a proxy to select for the binding sites which are likely to be functional. Indeed, TFBSs harbouring an essential function are under strong evolutionary constraint compared to neutrally evolving noncoding sequences, and will therefore display higher sequence conservation [41]. We have implemented this feature in TrawlerWeb whereby when input files are provided in BED format, corresponding genomic coordinates are matched against the reference genome selected. This provides the unique advantage for TrawlerWeb over other tools to display conservation scores for every instance of motifs discovered. To display conservation scores, PhastCons scores were downloaded from UCSC [35] and stored along with the chromosome sequences for each organism. For each instance of the identified motif in the input sequences, the average and maximum conservation score is calculated using the 
"bigWigOverAverage" tool provided in kentUtils [42] package from UCSC. Version 305 of kentUtils source is used as it offers "minMax" option to calculate the maximum value for conservation. The average and maximum conservation scores calculated from PhastCons scores are automatically displayed in the output (Fig. 3). Although this option is only available with the BED input option, it provides scientists the opportunity to filter for the evolutionarily conserved predicted binding sites for downstream biological validations.

\section{Conclusions}

Downstream analyses of DNA-binding genome-wide assays are paramount in elucidating a precise binding motif and in addition, identifying secondary proximal binding partners. As these experimental protocols become widely used, there is a need for easy access tools for scientists from all fields of research to perform de novo motif discovery. Here we have developed TrawlerWeb to allow fast and streamlined de novo motif discovery online, allowing direct search from NGS outputs using the BED format and generating an input-matched background. We have shown that TrawlerWeb performs faster than most popular motifs discovery tools, and robustly identifies the expected binding site. TrawlerWeb is primarily used to identify over-represented motifs in regions of DNA in ChIP-seq experiments for both transcription factors and histone marks. However, it can also be extended for identification of microRNA targets $[6,43]$, RNA-binding protein targets [44] or coexpression groups [45].

In conclusion, TrawlerWeb will appeal to a wide range of fields as the breadth of genome assemblies supported include commonly studied model organisms (Table 2 ). However, this list is currently limited to organisms for which conservation scores are available. To circumvent the need of a conservation score, users are still provided with the option to use FASTA formatted files with TrawlerWeb. Using FASTA input expands the range of analysis that can be performed with TrawlerWeb, by permitting analysis of datasets from partially sequenced genomes and from non-model organisms.

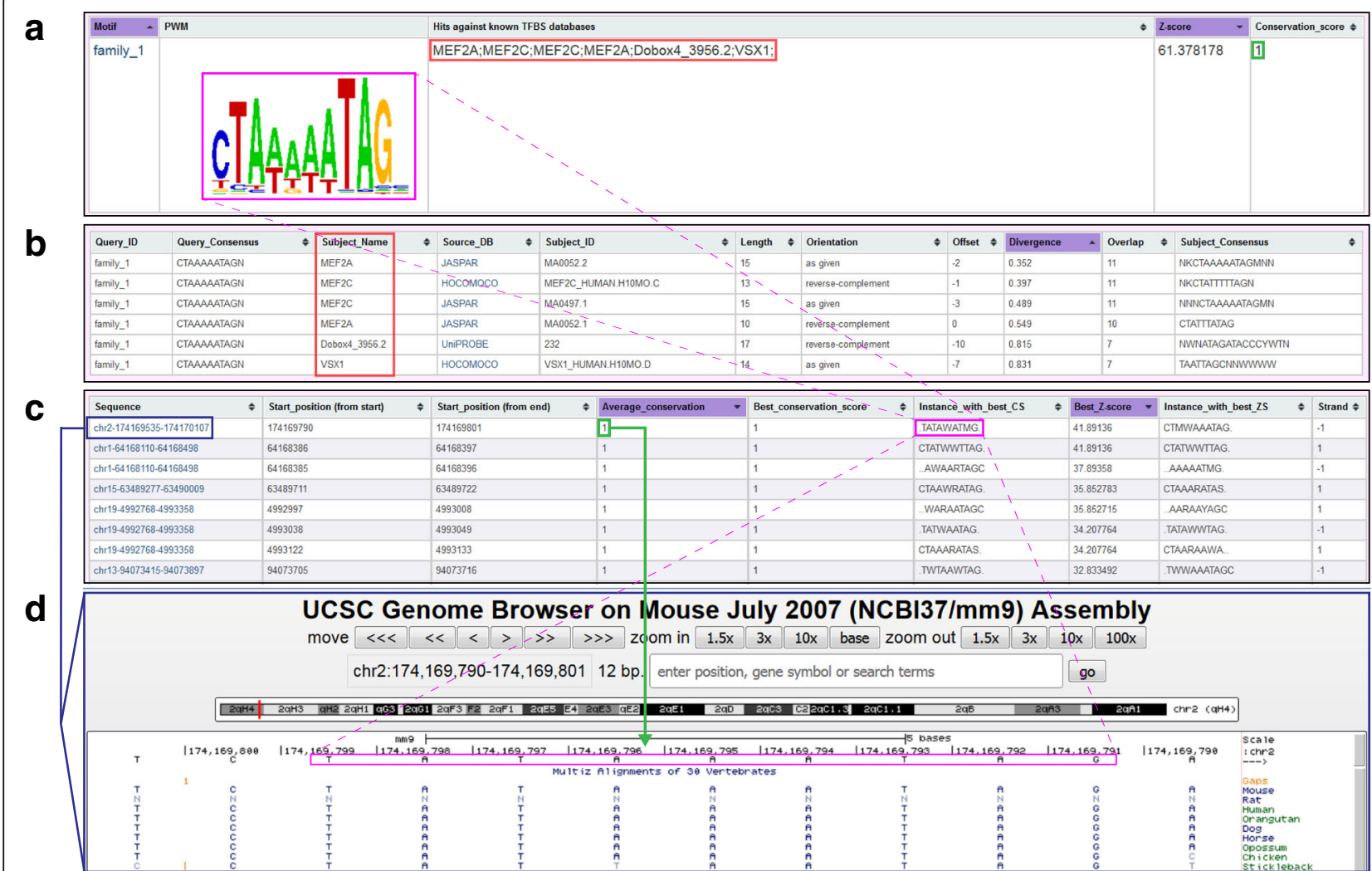

Fig. 3 TrawlerWeb output display with conservation scores and UCSC links. a TrawlerWeb displays the Position Weight Matrices (PWMs, pink box), Hits against known transcription factor binding site (TFBS) databases (red box), Z-scores of the discovered motifs, and the Conservation Score (green box). $\mathbf{b}$ Clicking on the PWM (pink box in (a)) directs the user to the list of putative matches (red box) and provides a direct link to the corresponding TFBS database entry. c Chromosomal positions of instances of the discovered motif (pink box) in the input peaks are also provided. Average and maximum conservation score (green box) will be available for each instance of the PWM. Clicking on the genomic region of interest (blue box) opens it in the UCSC Genome Browser (d) 


\section{Availability and requirements \\ Project name: TrawlerWeb.}

Project home page: https://trawler.erc.monash.edu.au/

Operating system(s): Platform independent.

Programming language: Java, Perl, HTML.

Other requirements: $\mathrm{N} / \mathrm{A}$.

License: The GNU General Public License (GPL) for

Trawler_standalone.

\section{Any restrictions to use by non-academics: N/A.}

\section{Additional files}

Additional file 1: Table S1. Details of the assessment of TrawlerWeb. Detailed table of Fig. 1b showing, for each ChIP experiment, the ability of individual programs to uncover the correct binding site in yeast. For each individual ChIP experiment, the success or failure of 8 different algorithms including TrawlerWeb is shown. The results from the 6 algorithms (Coverage, AlignACE, Kellis, mdscan, MEME, and MEME-c) were extracted from Harbison et al. 2004 [38]. The matching motifs found by TrawlerWeb are identical to that found by Trawler_standalone (detailed previously in Ettwiller et al. 2007 [5]). The results from RSAT were performed by this study, where the matching motifs found by RSAT were described in the last column. (XLSX $190 \mathrm{~kb}$ )

Additional file 2: Table S2. Details of motif occurrence comparison between web-based motif discovery software. The highest scoring motifs discovered in DREME, MEME, RSAT peak-motifs, and TrawlerWeb and their corresponding occurrences are illustrated here. For the highest scoring motif, the number of motif occurrences were expressed as a percentage of the total number of input sequences. *MEME-ChIP pre-processes submitted sequences longer than 100 by trimming them evenly from both ends to get the centered 100 bp sequence and discards trimmed sequences containing only Ns from repeat masking. ${ }^{* *}$ MEME motif discovery automatically limits the run to a randomly sampled 600 sequences to reduce run time. (XLSX $576 \mathrm{~kb}$ )

\section{Abbreviations}

ATAC: Assay for transposase-accessible chromatin; ChIP-seq: Chromatin immunoprecipitation followed by high-throughput sequencing; DamID: DNA Adenine methyltransferase identification; DREME: Discriminative regular expression motif elicitation; MCMC: Markov chain Monte Carlo; NGS: Nextgeneration sequencing; TFBS: Transcription factor binding site

\section{Acknowledgements}

We would like to thank Steve Androulakis from the Monash Bioinformatics Platform and Monash eResearch Centre for support in server space allocation. We would like to thank all members of the Monash Bioinformatics Platform and the Ramialison Laboratory for their support.

\section{Funding}

This work was supported by an Australian Research Council Discovery Project grant (DP1049980), a National Health and Medical Research Council/ Heart Foundation Career Development Fellowship (1049980), Sun Foundation to MR and UROP scholarships to LTD, MHHC, MJD and AT. The Australian Regenerative Medicine Institute is supported by grants from the State Government of Victoria and the Australian Government. This research was supported by use of the Nectar Research Cloud, a collaborative Australian research platform supported by the National Collaborative Research Infrastructure Strategy (NCRIS).

\section{Availability of data and materials}

The ChIP-seq datasets analysed in this study are available in the GEO repository under ascension numbers GSM1017643, GSE66225, GSM1377538, GSM878068, GSM1536045, [40, 46-49] and collected under https://github.com/RamialisonLab-ARMI/Trawler-2.0/tree/master/test_data/bedfiles.
The datasets generated during the study for testing purposes are available on GitHub at https://github.com/Ramialison-Lab-ARMI/Trawler-2.0/tree/ master/test_data.

\section{Authors' contributions}

LTD, MHHC, AT and MR designed and wrote the scripts to implement the new features of TrawlerWeb. JR operated the web server. LTD, MT, MHHC, JR, $B P, V T, A T, F B, G Q, H C, M J D, M P E, J C H, J S T, F J R, ~ H T N$ tested Trawler and provided intellectual input on TrawlerWeb's design. LTD, MT, BP, AT and MR wrote the manuscript with input from FB, MAB, DAJ, ERP and JEH. All authors read and approved the final manuscript.

Ethics approval and consent to participate

Not applicable

Consent for publication

Not applicable

\section{Competing interests}

The authors declare that they have no competing interests.

\section{Publisher's Note}

Springer Nature remains neutral with regard to jurisdictional claims in published maps and institutional affiliations.

\section{Author details \\ ${ }^{1}$ Australian Regenerative Medicine Institute, Systems Biology Institute Australia, Monash University, Clayton, VIC, Australia. ${ }^{2}$ eResearch, Monash University, Clayton, VIC, Australia. ${ }^{3}$ UC Santa Cruz Genomics Institute, University of California, Santa Cruz, CA, USA. ${ }^{4}$ Department of Biochemistry and Molecular Biology, Bio21 Institute and Cell Signalling Research Laboratories, The University of Melbourne, Melbourne, VIC, Australia. ${ }^{5} \mathrm{CNRS}$, Inserm, Institute of Biology Valrose, Université Côte d'Azur, Parc Valrose, Nice, France. ${ }^{6}$ School of Biomedical Sciences, The University of Queensland, QLD, Brisbane, Australia. 'Centre for Innate Immunity and Infectious Diseases, Hudson Institute of Medical Research, Monash University, Clayton, VIC, Australia. ${ }^{8}$ Department of Biochemistry and Molecular Biology, Monash University, Clayton, VIC, Australia. ${ }^{9}$ Faculty of Information Technology, Monash University, Clayton, VIC, Australia. ${ }^{10}$ Murdoch Children's Research Institute, The Royal Children's Hospital, Parkville, VIC, Australia. ${ }^{11}$ Department of Physiology, School of Biomedical Sciences, The University of Melbourne, Parkville, VIC, Australia.}

Received: 30 January 2018 Accepted: 27 March 2018

Published online: 05 April 2018

\section{References}

1. Consortium EP. An integrated encyclopedia of DNA elements in the human genome. Nature. 2012;489(7414):57-74.

2. Consortium F, the RP, Clst, Forrest AR, Kawaji H, Rehli M, Baillie JK, de Hoon MJ, Haberle V, Lassmann T, et al. A promoter-level mammalian expression atlas. Nature. 2014;507(7493):462-70.

3. Andersson R, Gebhard C, Miguel-Escalada I, Hoof I, Bornholdt J, Boyd M, Chen Y, Zhao X, Schmidl C, Suzuki T, et al. An atlas of active enhancers across human cell types and tissues. Nature. 2014;507(7493):455-61.

4. Roadmap Epigenomics C, Kundaje A, Meuleman W, Ernst J, Bilenky M, Yen A, Heravi-Moussavi A, Kheradpour P, Zhang Z, Wang J, et al. Integrative analysis of 111 reference human epigenomes. Nature. 2015;518(7539):317-30.

5. Ettwiller L, Paten B, Ramialison M, Birney E, Wittbrodt J. Trawler: de novo regulatory motif discovery pipeline for chromatin immunoprecipitation. Nat Methods. 2007:4(7):563-5.

6. Haudry Y, Ramialison M, Paten B, Wittbrodt J, Ettwiller L. Using Trawler_ standalone to discover overrepresented motifs in DNA and RNA sequences derived from various experiments including chromatin immunoprecipitation. Nat Protoc. 2010;5(2):323-34

7. Corradin O, Scacheri PC. Enhancer variants: evaluating functions in common disease. Genome medicine. 2014;6(10):85

8. Spitz F, Furlong EE. Transcription factors: from enhancer binding to developmental control. Nat Rev Genet. 2012;13(9):613-26.

9. Johnson DS, Mortazavi A, Myers RM, Wold B. Genome-wide mapping of in vivo protein-DNA interactions. Science. 2007;316(5830):1497-502. 
10. van Steensel B, Delrow J, Henikoff S. Chromatin profiling using targeted DNA adenine methyltransferase. Nat Genet. 2001;27(3):304-8.

11. Bouveret R, Waardenberg AJ, Schonrock N, Ramialison M, Doan T, de Jong D, Bondue A, Kaur G, Mohamed S, Fonoudi H, et al. NKX2-5 mutations causative for congenital heart disease retain functionality and are directed to hundreds of targets. elife. 2015;6:4. https://doi.org/10.7554/eLife.06942.

12. Buenrostro JD, Giresi PG, Zaba LC, Chang HY, Greenleaf WJ. Transposition of native chromatin for fast and sensitive epigenomic profiling of open chromatin, DNA-binding proteins and nucleosome position. Nat Methods. 2013;10(12):1213-8.

13. Zhou X, Li D, Zhang B, Lowdon RF, Rockweiler NB, Sears RL, Madden PA, Smirnov I, Costello JF, Wang T. Epigenomic annotation of genetic variants using the roadmap epigenome browser. Nat Biotechnol. 2015;33(4):345-6.

14. Romanoski CE, Glass CK, Stunnenberg HG, Wilson L, Almouzni G. Epigenomics: roadmap for regulation. Nature. 2015;518(7539):314-6.

15. Budden DM, Hurley DG, Crampin EJ. Predictive modelling of gene expression from transcriptional regulatory elements. Brief Bioinform. 2015; 16(4):616-28.

16. Suryamohan $\mathrm{K}$, Halfon MS. Identifying transcriptional cis-regulatory modules in animal genomes. Wiley Interdiscip Rev Dev Biol. 2015;4(2):59-84.

17. Kuttippurathu L, Hsing M, Liu Y, Schmidt B, Maskell DL, Lee K, He A, Pu WT, Kong SW. CompleteMOTIFs: DNA motif discovery platform for transcription factor binding experiments. Bioinformatics. 2011;27(5):715-7.

18. Machanick P, Bailey TL. MEME-ChIP: motif analysis of large DNA datasets. Bioinformatics. 2011;27(12):1696-7.

19. Sharov AA, Ko MS. Exhaustive search for over-represented DNA sequence motifs with CisFinder. DNA Res. 2009;16(5):261-73.

20. Thomas-Chollier M, Herrmann C, Defrance M, Sand O, Thieffry D, van Helden J. RSAT peak-motifs: motif analysis in full-size ChIP-seq datasets. Nucleic Acids Res. 2012;40(4):e31.

21. Zambelli F, Pesole G, Pavesi G. PscanChIP: finding over-represented transcription factor-binding site motifs and their correlations in sequences from ChIP-Seq experiments. Nucleic Acids Res. 2013;41(Web Server issue): W535-43. https://doi.org/10.1093/nar/gkt448. Epub 2013 Jun 7

22. Zhou J, Troyanskaya OG. Predicting effects of noncoding variants with deep learning-based sequence model. Nat Methods. 2015;12(10):931-4.

23. Alipanahi B, Delong A, Weirauch MT, Frey BJ. Predicting the sequence specificities of DNA- and RNA-binding proteins by deep learning. Nat Biotech. 2015;33(8):831-8.

24. Afgan $E$, Baker D, van den Beek M, Blankenberg D, Bouvier D, Cech M, Chilton J, Clements D, Coraor N, Eberhard C, et al. The galaxy platform for accessible, reproducible and collaborative biomedical analyses: 2016 update. Nucleic Acids Res. 2016;44(W1):W3-W10.

25. Ambrosini G, Dreos R, Kumar S, Bucher P. The ChIP-Seq tools and web server: a resource for analyzing ChIP-seq and other types of genomic data. BMC Genomics. 2016;17(1):938.

26. Aken BL, Ayling S, Barrell D, Clarke L, Curwen V, Fairley S, Fernandez Banet J, Billis K, Garcia Giron C, Hourlier T et al: The Ensembl gene annotation system. Database (Oxford). 2016;2016. doi: https://doi.org/10.1093/database/ baw093. Print 2016.

27. Quinlan AR, Hall IM. BEDTools: a flexible suite of utilities for comparing genomic features. Bioinformatics. 2010;26(6):841-2.

28. Fleming JD, Pavesi G, Benatti P, Imbriano C, Mantovani R, Struhl K. NF-Y coassociates with FOS at promoters, enhancers, repetitive elements, and inactive chromatin regions, and is stereo-positioned with growthcontrolling transcription factors. Genome Res. 2013;23(8):1195-209.

29. Vavouri T, Lehner B. Human genes with CpG island promoters have a distinct transcription-associated chromatin organization. Genome Biol. 2012; 13(11):R110.

30. Kinsella RJ, Kahari A, Haider S, Zamora J, Proctor G, Spudich G, Almeida-King J, Staines D, Derwent P, Kerhornou A, et al. Ensembl BioMarts: a hub for data retrieval across taxonomic space. Database (Oxford). 2011;2011:bar030.

31. Hume MA, Barrera LA, Gisselbrecht SS, Bulyk ML. UniPROBE, update 2015: new tools and content for the online database of protein-binding microarray data on protein-DNA interactions. Nucleic Acids Res. 2015; 43(Database issue):D117-22.

32. Mathelier A, Zhao X, Zhang AW, Parcy F, Worsley-Hunt R, Arenillas DJ, Buchman S, Chen CY, Chou A, lenasescu H, et al. JASPAR 2014: an extensively expanded and updated open-access database of transcription factor binding profiles. Nucleic Acids Res. 2014;42(Database issue):D142-7.
33. Kulakovskiy IV, Medvedeva YA, Schaefer U, Kasianov AS, Vorontsov IE, Bajic VB, Makeev VJ. HOCOMOCO: a comprehensive collection of human transcription factor binding sites models. Nucleic Acids Res. 2013; 41(Database issue):D195-202.

34. Kulakovskiy IV, Vorontsov IE, Yevshin IS, Soboleva AV, Kasianov AS, Ashoor H, Ba-Alawi W, Bajic VB, Medvedeva YA, Kolpakov FA, et al. HOCOMOCO: expansion and enhancement of the collection of transcription factor binding sites models. Nucleic Acids Res. 2016;44(D1):D116-25.

35. Kent WJ, Sugnet CW, Furey TS, Roskin KM, Pringle TH, Zahler AM, Haussler D. The human genome browser at UCSC. Genome Res. 2002;12(6):996-1006.

36. Thomas-Chollier M, Darbo E, Herrmann C, Defrance M, Thieffry D, van Helden J. A complete workflow for the analysis of full-size ChIP-seq (and similar) data sets using peak-motifs. Nat Protoc. 2012;7(8):1551-68.

37. Bailey TL. DREME: motif discovery in transcription factor ChIP-seq data. Bioinformatics. 2011:27(12):1653-9.

38. Harbison CT, Gordon DB, Lee TI, Rinaldi NJ, Macisaac KD, Danford TW, Hannett NM, Tagne JB, Reynolds DB, Yoo J, et al. Transcriptional regulatory code of a eukaryotic genome. Nature. 2004;431(7004):99-104.

39. Mahony S, Benos PV. STAMP: a web tool for exploring DNA-binding motif similarities. Nucleic Acids Res. 2007;35(Web Server issue):W253-8. Epub 2007 May 3

40. Sulahian R, Chen J, Arany Z, Jadhav U, Peng S, Rustgi AK, Bass AJ, Srivastava A, Hornick JL, Shivdasani RA. SOX15 governs transcription in human stratified epithelia and a subset of esophageal adenocarcinomas. Cell Mol Gastroenterol Hepatol. 2015;1 (6):598-609.e6.

41. Woolfe A, Goodson M, Goode DK, Snell P, McEwen GK, Vavouri T, Smith SF, North P, Callaway H, Kelly K, et al. Highly conserved non-coding sequences are associated with vertebrate development. PLoS Biol. 2005;3(1):e7.

42. Kent WJ, Zweig AS, Barber G, Hinrichs AS, Karolchik D. BigWig and BigBed: enabling browsing of large distributed datasets. Bioinformatics. 2010;26(17): 2204-7.

43. Linsley PS, Schelter J, Burchard J, Kibukawa M, Martin MM, Bartz SR, Johnson JM, Cummins JM, Raymond CK, Dai H, et al. Transcripts targeted by the microRNA-16 family cooperatively regulate cell cycle progression. Mol Cell Biol. 2007:27(6):2240-52

44. Konig J, Zarnack K, Luscombe NM, Ule J. Protein-RNA interactions: new genomic technologies and perspectives. Nat Rev Genet. 2012;13(2):77-83.

45. Ramialison M, Reinhardt R, Henrich T, Wittbrodt B, Kellner T, Lowy CM, Wittbrodt J. Cis-regulatory properties of medaka synexpression groups. Development. 2012;139(5):917-28.

46. Houles T, Rodier G, Le Cam L, Sardet C, Kirsh O. Description of an optimized ChIP-seq analysis pipeline dedicated to genome wide identification of E4F1 binding sites in primary and transformed MEFs. Genom Data. 2015;5:368-70. https://doi.org/10.1016/j.gdata.2015.07.004. eCollection 2015 Sep

47. Huang W, Perez-Garcia P, Pokhilko A, Millar AJ, Antoshechkin I, Riechmann $I L$, Mas P. Mapping the core of the Arabidopsis circadian clock defines the network structure of the oscillator. Science. 2012;336(6077):75-9.

48. Skalska L, Stojnic R, Li J, Fischer B, Cerda-Moya G, Sakai H, Tajbakhsh S, Russell S, Adryan B, Bray SJ. Chromatin signatures at notch-regulated enhancers reveal large-scale changes in H3K56ac upon activation. EMBO J. 2015;34(14):1889-904.

49. Winata $\mathrm{CL}$, Kondrychyn I, Kumar V, Srinivasan KG, Orlov Y, Ravishankar A Prabhakar S, Stanton LW, Korzh V, Mathavan S. Genome wide analysis reveals Zic3 interaction with distal regulatory elements of stage specific developmental genes in zebrafish. PLoS Genet. 2013;9(10):e1003852. 